and mass, with more concentric remodelling, lower systolic (LV ejection fraction, longitudinal and circumferential global peak systolic strain) and diastolic function (longitudinal and circumferential peak early diastolic strain rates) than females. Late gadolinium enhancement (LGE) was more prevalent $(51.1 \%$ vs. $34.1 \%, \mathrm{p}=0.038)$ and extent of LGE was also higher in males, as was Syndecan-4 and MMP-3 levels. Extracellular volume (ECV) was marginally higher in female patients. Stress and rest myocardial blood flow (MBF) were significantly lower in males, with no difference in myocardial perfusion reserve.

Conclusions Male patients with AS have more concentric remodelling, worse cardiac function and more fibrosis than females, with biomarkers associated with fibrosis being significantly higher as well, for a similar degree of AS.

\begin{tabular}{llll}
\multicolumn{4}{l}{ Abstract 129 Table 1} \\
\hline & Male $(\mathrm{n}=133)$ & Female $(\mathrm{n}=41)$ & $\mathrm{p}=\mathrm{value}$ \\
\hline Age (years) & $67.3 \pm 12.64$ & $62.9 \pm 15.08$ & $0.042^{*}$ \\
Mean PG (mmHg) & $34.5 \pm 12.05$ & $38.0 \pm 13.66$ & 0.491 \\
AVAl $\left(\mathrm{cm}^{2} / \mathrm{m}^{2}\right)$ & $0.58 \pm 0.14$ & $0.55 \pm 0.15$ & 0.595 \\
LVEDVI $\left(\mathrm{ml} / \mathrm{m}^{2}\right)$ & $90.00 \pm 18.67$ & $79.74 \pm 14.50$ & $<0.001^{*}$ \\
LVEF $(\%)$ & $55.9 \pm 4.84$ & $59.2 \pm 4.49$ & $<0.001^{*}$ \\
LVMI $\left(\mathrm{g} / \mathrm{m}^{2}\right)$ & $60.54 \pm 13.70$ & $48.45 \pm 9.74$ & $<0.001^{*}$ \\
LV mass $/ \mathrm{volume}(\mathrm{g} / \mathrm{ml})$ & $0.68 \pm 0.11$ & $0.61 \pm 0.11$ & $<0.001^{*}$ \\
PSS $(\%)$ & $-17.85 \pm 2.80$ & $-20.52 \pm 2.81$ & $<0.001^{*}$ \\
PSS $_{C}(\%)$ & $-27.64 \pm 4.83$ & $-29.56 \pm 3.74$ & $0.002^{*}$ \\
\hline
\end{tabular}

\begin{tabular}{llll} 
Abstract 129 Table 1 & & \\
\hline & Male $(\mathbf{n}=133)$ & Female $(\mathbf{n}=41)$ & p-value \\
\hline Stress MBF $(\mathrm{m} / \mathrm{mm} / \mathrm{g})$ & $2.09 \pm 0.66$ & $2.39 \pm 0.80$ & $0.009^{*}$ \\
Rest MBF $(\mathrm{m} / \mathrm{min} / \mathrm{g})$ & $0.93 \pm 0.21$ & $1.14 \pm 0.36$ & $<0.001^{*}$ \\
Global MPR & $2.29 \pm 0.70$ & $2.18 \pm 0.70$ & 0.449 \\
$\%$ LGE (\%) & $4.6 \pm 3.87$ & $2.9 \pm 3.06$ & $0.001^{*}$ \\
ECV (\%) & $24.57 \pm 2.54$ & $25.64 \pm 1.85$ & $0.007^{*}$ \\
Log10.Syndecan-4 (pg/ml) & $2.33[$ IIQR 0.72] & $2.14[$ [IQR 2.39] & $0.044^{*}$ \\
Log10.MMP-3 (pg/ml) & $4.41[$ IIQR 0.88] & $4.24[$ [IQR 0.71] & $0.041^{*}$ \\
\hline
\end{tabular}

\section{THE CONVERGENCE AND DIVERGENCE OF MOLECULAR PATHWAYS IN LV HYPERTROPHY DEFINED BY ECG VOLTAGE VERSUS LV MASS IN PATIENTS WITH AORTIC STENOSIS}

${ }^{1}$ Jiangting Hu, ${ }^{2}$ John Pepper, ${ }^{3}$ Francesco Pezzella, ${ }^{4}$ Kevin Gatter, ${ }^{5} \mathrm{Xu}$ Yu Jin*. ${ }^{1}$ Nuffield Division of clinical lab Sciences, Radcliffe Department of Medician, University of Oxford; ${ }^{2}$ Academic Cardiac Surgery, Royal Brompton Hospital; ${ }^{3}$ Nuffield Division of Clinical Lab Sciences, Radcliffe Department of Medicine, University of Oxford; ${ }^{4}$ Nuffield Division of Cliinical Lab Sciences, Redcliffe Department of Medician, University of Oxford; ${ }^{5}$ Department of Cardiothoracic Surgery, John Radcliffe Hospital

\subsection{6/heartjnl-2017-311726.129}

Background Left ventricular hypertrophy (LVH) assessment by ECG voltage or image based mass volume is commonly used in clinical practice and research. Our previous study of LVH regression after AVR for AS demonstrated a more complete regression in ECG voltage than in LV mass, thus indicated that ECG and Echo could be quantifying different aspects of
LVH. In this study, we used gene expression profiling to examine whether different molecular pathways are involved in LV hypertrophy defined by ECG voltage or Echo LV mass.

Material and Methods We studied 17 patients with aortic stenosis, aged $73 \pm 8.5$ years with 12 males. Gene expression profiling of LV myocardium biopsy during AVR was carried out using Stanford Human Exonic Evidence Based Oligonucleotide (HEEBO) array. Using ECG QRS voltage cut-off $3.0 \mathrm{mv}, 8$ patients had LVH (ECG-LVH) and 9 had not (ECG-Norm ). Using Echo LV mass index $125 \mathrm{~g} / \mathrm{m} 2$ for man and $105 \mathrm{~g} / \mathrm{m} 2$ for woman, 9 cases had LVH (Echo-LVH) and 8 had not (Echo-Norm). The gene expression profiling comparisons were carried out between ECG-LVH vs. ECG-Norm as well as Echo-LVH vs. Echo-Norm, respectively, by parametric permutative (permutation times $=1000$ ) t-test using the $p$ value cut-off 0.01. Further gene functional annotation clusters were performed using Database for Annotation, Visualisation and Integrated Discovery (DAVID, NIH) and pathway analysed by GeneSpring GX10.

Results There are 163 genes significantly differentially expressed (DEGs) between ECG-LVH and ECG-Norm. Among which, 81 genes were up-regulated and 82 genes down-regulated in ECG-LVH. Between Echo-LVH and Echo-Norm, 256 DEGs were identified, of which 141 genes were up and 115 genes were down regulated in Echo-LVH. The Direct Interaction Network of 163 DEGs in ECG-LVH revealed several connected island hubs with genes of HGF, CDK5RAP2, FYN (up-regulated) and IL6, PTBP2 and IRF1 (down-regulated). Of 257 DEGs in Echo-LVH, there were more abundant islandlike connexions. The hubs of the connexion include PTEN, GNL3, AREG, ADAM17 and PPP1CA (up-regulated) and DCTN6, TWIST1 and ITGAM (down regulated). Further data mining and analysis of the two DEGs lists using DAVID and the Significantly Enriched Core Pathways analysis has demonstrated that the DEGs of ECG-LVH and ECHO-LVH have similar regulation directions in pathways/annotation clustering, including transcription regulation, nucleotide binding, zinc finger, focal adhesion. In addition, ECG-LVH has down regulation in wounding healing/inflammatory/cytokines pathway indicating a lower interstitial fibrosis activity compared with ECG-Norm; In Echo-LVH, mitochondrial protein importing, metalloprotease/ECM remodelling and cell division pathways were up-regulated compared to Echo-Norm.

Conclusions Cardiac hypertrophy diagnosed by ECG voltage or Echo muscle mass share many common molecular pathways that have been implicated in LVH. The unique pathways associated with ECG voltage based LVH imply a predominant hypertrophy of myocytes; in contrast, Echo mass based LVH involves the unique pathways of myocardial energy and ECM remodelling. The convergence and divergence of underlying molecular pathways between ECG based and Echo based LVH suggest the two methods may provide complimentary clinical value in the assessment of $\mathrm{LVH}$ and its response to treatment.

\section{LEFT VENTRICULAR INDEXED MASS ASSOCIATED WITH VENTRICULAR ARRHYTHMIAS IN PATIENTS WITH HYPERTROPHIC CARDIOMYOPATHY - A TERTIARY CENTRE MRI REGISTRY}

${ }^{1}$ Habib Khan*, ${ }^{1}$ Konstantinos Somarakis, ${ }^{2}$ Andrew Thain, ${ }^{1}$ Ayman Al-Atta, ${ }^{1}$ Thomas Mathew. ${ }^{1}$ Nottingham City Hospital; ${ }^{2}$ London Health Sciences Centre

10.1136/heartjnl-2017-311726.130 
Background Hypertrophic cardiomyopathy (HCM) is a common inherited cardiac condition. Multiple factors have been identified towards high risk of sudden cardiac death (SCD) as outlined in the ESC guidelines. $\mathrm{LV}$ wall thickness $\left(\mathrm{LV}_{\mathrm{wt}}\right)$ is an identified risk. Left ventricular mass indexed to body surface area $\left(\mathrm{LV}_{\mathrm{IBSA}}\right)$ increases with wall thickness and may predict risk not currently utilised in the risk score.

Objectives 1. Compare differences in $\mathrm{LV}_{\mathrm{wt}}$ using transthoracic echocardiogram(TTE) and MRI and the effect on ESC risk score.

2. Observe for association of $\operatorname{LV}_{\text {IBSA }}$ and incidence of nonsustained ventricular tachycardia(NSVT) either on 24 hour holter monitor or following ICD insertion.

Methods We retrospectively reviewed patients between January 2010 to July 2015 who were confirmed to have HCM on MRI. ESC Risk was calculated using LV TTE and MRI LV ${ }_{w t}$. $L_{\text {IBSA }}$ was calculated from MRI images and compared with incidence of VA on holter monitors. Patients who received ICD had follow up and VA incidence recorded.

Results 103 patients with confirmed HCM were identified with median age of 60 (range 15-87). Non sustained VT (NSVT) was recorded in $20(19.4 \%)$ patients while 16 patients had missing or no record of holter. Primary prevention ICDs (ICD ${ }_{1 *}$ ) were inserted in 20 (19.4\%) patients. MRI identified a higher absolute $\mathrm{LV}_{\mathrm{wt}}$ compared to TTE in $68.9 \%$ of patients. This lead to an increase in ESC risk score from low risk to high risk in $5 \%$ of the patients (ESC score $>4)$. LV IBSA $_{\text {Ias }}$ higher in the patients with holter positive for VA (mean $109.7 \mathrm{~g} / \mathrm{m}^{2}, 95 \%$ CI [92.8, 126.6] vs $89.8 \mathrm{~g}$ / $\mathrm{m}^{2}$, 95\% CI [83.2, 96.4], $\left.\mathrm{p}=<0.05\right)$. Patients with ICD $(n=20)$ were followed up for 52.6 months \pm 5.8 months. One patient with ICD $1^{*}$ had VA detected after 7 months of ICD insertion and treated successfully with 1 anti-tachycardia pacing algorithm.

Conclusion The detection of HCM through use of MRI allows for earlier diagnosis of patients and provides for accurate and reproducible measurements of $L_{\mathrm{wt}}$ and $\operatorname{LV}_{\mathrm{IBSA}} \mathrm{x}$ as opposed to TTE. In our retrospective study it is suggested that higher $L_{\text {IBSA }}$ is related to increase incidence of VA. If $\mathrm{LV}_{\text {IBSA }}$ derived from MRI were applied to ESC guidelines, this could result in more patients ICD ${ }_{1 *}$. We feel this should be explored with larger studies to see if $\mathrm{LV}_{\mathrm{IBSA}}$ is an independent risk factor for SCD.

\section{THE IMPORTANCE OF CONTRACTILE RESERVE WHEN ASSESSING ASYMPTOMATIC PATIENTS WITH AORTIC STENOSIS}

${ }^{1}$ Jet van Zalen*, ${ }^{2}$ Sveeta Badiani, ${ }^{1}$ Lesley Hart, ${ }^{3}$ Gary Brickley, ${ }^{3}$ Louisa Beale, ${ }^{1}$ Andrew Marshall, ${ }^{1}$ Nikhil Patel, ${ }^{2}$ Guy Lloyd. ${ }^{1}$ East Sussex Healthcare NHS Trust; ${ }^{2}$ Barts Heart centre; ${ }^{3}$ University of Brighton

\subsection{6/heartjnl-2017-311726.131}

Asymptomatic patients may exhibit symptoms during objective exercise testing, but whether symptoms are due to the obstructivity of the valve (typified by the mean gradient) or underlying ventricular function remains unknown. While the mean gradient is an easy parameter to measure no consensus about the measurement of contractile reserve exists. Longitudinal abnormalities may occur in the presence of a normal ejection fraction and the augmentation of these parameters is poorly described. The aim of this study was to examine the echocardiographic predictors of exercise ability during cardiopulmonary exercise testing combined with stress echocardiography.

24 asymptomatic patients with moderate to severe or severe aortic stenosis and preserved ejection fraction underwent stress echocardiography with simultaneous cardiopulmonary exercise testing. The primary assessment of exercise ability was $\mathrm{VO}_{2}$ peak. Echocardiography was measured at rest and during maximal exercise (defined as RER $>1$ )

$\mathrm{VO}_{2}$ peak showed a poor relationship with conventional resting parameters of severity including peak velocity $(\mathrm{rho}=0.07 ; \mathrm{p}=\mathrm{ns})$, mean pressure gradient $(\mathrm{rho}=0.3 ; \mathrm{p}=\mathrm{ns})$, AVA $(\mathrm{rho}=0.4 ; \mathrm{p}=\mathrm{ns})$, dimensionless index $(\mathrm{rho}=0.05 ; \mathrm{p}=\mathrm{ns})$, resting systolic function (by $\mathrm{EF}(\mathrm{rho}=-0.18 ; \mathrm{p}=\mathrm{ns})$ and TDI $(\mathrm{rho}=0.39 ; \mathrm{p}=\mathrm{ns})$. During exercise systolic augmentation had a good relationship with exercise ability (rho $=0.77$; $\mathrm{p}<0.0001)$ but the relationship with exercise mean gradient was weaker $(r h o=0.57 ; p=0.005)$ and there was no relationship with exercise LVEF ( $r h o=0.18 ; \mathrm{p}=\mathrm{ns}$ ).

Longitudinal systolic function during peak exercise is the strongest predictor of exercise ability when compared to conventional measures of severity of aortic stenosis.

\section{CARDIOPULMONARY EXERCISE TESTING: DOES ETHNICITY MATTER?}

Keerthi Prakash*, Stathis Papatheodorou, Bode Ensam, Chris Miles, Saeed Azizi, Paulo Bulleros, Zephryn Fanton, Aneil Malhotra, Andrew D'Silva, Gherardo Finocchiaro, Traceey Keteepe-Arachi, Maite Tome, Nicholas Bunce, Juan-Carlos Kaski, Michael Papadakis, Sanjay Sharma. St George's, University of London

\subsection{6/heartjnl-2017-311726.132}

Introduction Hypertrophic cardiomyopathy (HCM) is the largest cause of sudden cardiac death in athletes. Differentiation between HCM and athletic cardiac adaptation is not always straightforward. Cardiopulmonary exercise testing (CPET) is useful in this context, with a peak $\mathrm{VO}_{2}>120 \%$ predicted commonly used to differentiate athletes with HCM from those with physiological left ventricular hypertrophy. This value however is derived from a predominantly white population. Differences with ethnicity have been well documented on the ECG and echocardiogram of both athletes and individuals with HCM, however, ethnic differences in their physiology have not been well investigated to date.

Purpose To assess if there is a significant difference on CPET in HCM patients of black and white ethnicity.

Methods Cardiopulmonary exercise testing data was prospectively and retrospectively analysed from a cohort of 49 sedentary HCM patients assessed in a quaternary referral centre (36 white, 38 male; aged 15-65 years). Inclusion criteria: HCM patients of black or white ethnicity, NYHA 1, resting LVOT gradient $<40 \mathrm{mmHg}$, no ICD in-situ and having completed a maximal CPET (defined as: R 1.1 and test terminated due to breathlessness/muscular fatigue). 